\title{
GDR cross sections updated in the IAEA-CRP
}

Hiroaki Utsunomiya ${ }^{1, *}$, Ioana Gheorghe ${ }^{2}$, Dan M. Filipescu ${ }^{2}$, Konstantin Stopani ${ }^{3}$, Sergey Belyshev ${ }^{4}$, Hongwei Wang $^{5}$, Gongtao Fan ${ }^{5}$, Takashi Ariizumi ${ }^{1}$, Yiu-Wing Lui ${ }^{6}$, Dmytro Symochko ${ }^{7}$, Mateusz Krzysiek ${ }^{8}$, Shuji Miyamoto ${ }^{9}$, Stephane Goriely ${ }^{10}$, and Toshihiko Kawano ${ }^{11}$

${ }^{1}$ Department of Physics, Konan University, Kobe, Japan

${ }^{2}$ Horia Hulubei National Institute for Physics and Nuclear Engineering (IFIN-HH), Bucharest-Magurele, Romania

${ }^{3}$ Department of Physics, Lomonosov Moscow State University, Russia

${ }^{4}$ Lomonosov Moscow State University, Skobeltsyn Institute of Nuclear Physics, Russia

${ }^{5}$ Shanghai Institute of Applied Physics, China

${ }^{6}$ Cyclotron Institute, Texas A \& M university, USA

${ }^{7}$ Technical University Darmstadt, Germany

${ }^{8}$ Institute of Nuclear Physics Polish Academy of Sciences, Krakow, Poland

${ }^{9}$ Laboratory of Advanced Science and Technology for Industry, University of Hyogo, Japan

${ }^{10}$ Institut dfAstronomie et dfAstrophysique, Université Libre de Bruxelles, Belgium

${ }^{11}$ Los Alamos National Laboratory, USA

Abstract. The Coordinated Research Project (CRP) with the code F41032 has been launched by the International Atomic Energy Agency (IAEA) in 2016 as a 5-year project with the scientific goal being two-fold: 1) updating the 2000 photonuclear data library and 2) constructing a reference database of photon strength functions. We report the experimental technique and methodology used for the former goal and selected giant-dipole resonance (GDR) data updated in the IAEA-CRP.

\section{IAEA-CRP}

The last IAEA photonuclear data library (IAEATECDOC-1178) [1] was published in 2000 which has compiled and evaluated the photoneutron data of 164 isotopes of 48 elements from ${ }^{2} \mathrm{H}$ to ${ }^{241} \mathrm{Pu}$. It is well known that there is a long-standing discrepancy between the Livermore and Saclay data of partial photoneutron cross sections for 19 nuclei from ${ }^{51} \mathrm{~V}$ to ${ }^{238} \mathrm{U}$ among 42 nuclei commonly measured at Livermore and Saclay [2]. Although there is a general tendency that the Saclay data of $(\gamma, 1 n)$ cross section is larger than the Livermore data and vice versa in the $(\gamma, 2 n)$ cross section, the ratio of energy-integrated cross sections between Livermore and Saclay scatters so largely that the discrepancy cannot be resolved in a systematic way [2,3]. Due to this fact, it was requested in the IAEA-CRP $[4,5]$ to acquire new data with improved accuracy that help to resolve the discrepancy. We have newly obtained photoneutron data for 11 nuclei, ${ }^{9} \mathrm{Be},{ }^{59} \mathrm{Co},{ }^{89} \mathrm{Y},{ }^{103} \mathrm{Rh},{ }^{139} \mathrm{La},{ }^{159} \mathrm{~Tb},{ }^{165} \mathrm{Ho},{ }^{169} \mathrm{Tm}$, ${ }^{181} \mathrm{Ta},{ }^{197} \mathrm{Au}$, and ${ }^{209} \mathrm{Bi}$ in the PHOENIX collaboration performed in the $\gamma$-ray beam line of the NewSUBARU synchrotron radiation facility.

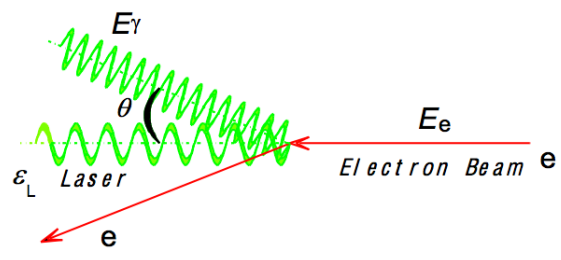

Figure 1. (Color online) Laser Compton scattering.

\section{Experimental technique and methodology}

The PHOENIX collaboration is featured with two experimental factors that assure improved accuracy in measuring photoneutron cross sections: the $\gamma$-ray beam and methodology of neutron-multiplicity sorting.

\subsection{Laser Compton-scattered $\gamma$-ray beam}

Quasi-monochromatic $\gamma$-ray beams were produced in collisions of laser photons with relativistic electrons in a straight line of the NewSUBARU storage ring. Figure 1 depicts the laser Compton scattering. Fig. 2 shows the $\gamma$ ray beam line of the NewSUBARU facility. Electrons at $974 \mathrm{MeV}$ in nominal energy were injected into the storage ring from a linear accelerator and either decelerated to $0.55 \mathrm{GeV}$ or accelerated to $1.46 \mathrm{GeV}$. Two lasers at

*e-mail: hiro@konan-u.ac.jp 
the wave length of $1064 \mathrm{~nm}$ (INAZUMA laser) and 532 $\mathrm{nm}$ (Talon laser) were used: the former for measuring $(\gamma, 1 n)$ cross sections between $1 n$ and $2 n$ thresholds and the latter for measuring $(\gamma, i n)$ cross sections with the neutron multiplicity $i=1-4$ between $2 n$ and $5 n$ thresholds. The laser Compton-scattered (LCS) $\gamma$-ray beam is energytunable by changing the electron beam energy; a laserphoton beam is energy-boosted by a factor of $4.6 \times 10^{6}$ to $3.3 \times 10^{7}$ and converted to a $\gamma$-ray beam in the $\mathrm{MeV}$ range.

The LCS $\gamma$-ray beam is accompanied by synchrotron radiation whose energy is significantly lower than the energy of the maximum $\gamma$-ray yield; the synchrotron radiation did not interfere photoneutron cross section measurements. This is essentially different from background $\gamma$ rays (positron bremsstrahlung) produced in the positron annihilation in flight [6]. The contribution from the positron bremsstrahlung was a major concern, requiring the second measurement by replacing the positron beam with an electron beam to subtract the contribution. In the measurement with the LCS $\gamma$-ray beam, the laser was turned on for 80 $\mathrm{ms}$ and off for $20 \mathrm{~ms}$ in every $100 \mathrm{~ms}$ by gating the laser with transistor-transistor logic signals with $80 \mathrm{~ms}$ width at $10 \mathrm{~Hz}$. Backgroud neutrons of the accelerator and cosmicray origin were measured during the laser off.

\subsection{Energy calibration of the $\gamma$-ray beam}

Energy calibration of tens of $\mathrm{MeV} \gamma$ rays with the standard $\gamma$-ray sources like ${ }^{137} \mathrm{Cs}$ and ${ }^{60} \mathrm{Co}$ lacks accuracy because of an uncertain extrapolation of a calibration curve to the high-energy region. The energy of the LCS $\gamma$-ray beam becomes maximum in the head-on collision corresponding to scattering at $\theta=0$ degree in Fig. 1, where the maximum energy is determined solely by the electron beam energy. Based on the kinematics of the laser Compton scattering, it is possible to determine the LCS $\gamma$-ray beam energy from the electron beam energy.

We produced low-energy LCS $\gamma$ rays in a few MeV region with a grating-fixed $\mathrm{CO}_{2}$ laser with the wave length of $10.5915 \mu \mathrm{m} \pm 3$ Åand measured with a high-purity germanium detector that was energy-calibrated with the standard $\gamma$-ray sources. Electron beam energies were determined by reproducing the high-energy edge of the LCS $\gamma$-ray beams by Monte Carlo simulations on the order of $10^{-5}[7,8]$. The reproducibility of the electron beam energy is excellent in every injection of an electron beam from the linear accelerator into the storage ring followed by the deceleration down to $0.55 \mathrm{GeV}$ [7] and acceleration up to 1.46 $\mathrm{GeV}$ [8] due to an automated control of the electron beamoptics parameters.

\subsection{Energy profile of the $\gamma$-ray beam}

Energy profiles of high-energy LCS $\gamma$-ray beams were determined by best reproducing response functions of a 3.5" $\times 4.0^{\prime \prime} \mathrm{LaBr}_{3}(\mathrm{Ce})$ detector to the $\gamma$-ray beams by Monte Carlo simulations with a GEANT4 code that incorporates the kinematics of the laser Compton scattering and transportation of the $\gamma$-ray beam through two $\mathrm{Pb}$ collimators,
$\mathrm{C} 1$ of $3 \mathrm{~mm}$ diameter and $\mathrm{C} 2$ of $2 \mathrm{~mm}$ diameter in Fig. 2. The electron-beam energy was fixed at the calibrated energy in the simulation. The electron beam has a waist at the most effective collision point ( $\mathrm{P} 2$ for the INAZUMA and Talon lasers and $\mathrm{P} 1$ for the $\mathrm{CO}_{2}$ laser). The size of the waist, which is much smaller than the size of the laserphoton beam, is $0.3 \mathrm{~mm}$ horizontally and $0.18 \mathrm{~mm}$ vertically at P2 [9].

By the GEANT4 simulation, the best-fit values of the experimentally unknown parameters involved in the kinematics, the horizontal and vertical electron beam emittances, were obtained with which the energy profile of the $\gamma$-ray beam was determined. Figure 3 shows experimental response function data at energies from 6 to $39 \mathrm{MeV}$, bestfit simulations, and energy profiles of the incident LCS $\gamma$ ray beam. The energy spread was $1-3 \%$ in the full width at half maximum. The energy spread depends on the size of the collimators which confine scattering angles around $\theta=0$ degree (Fig. 1) and the electron beam emittance as well as the energy resolution of the electron beam which is $10^{-4}$. There is a limit on the $\mathrm{C} 2$ collimator size around $1 \mathrm{~mm}$ diameter below which the electron-beam emittance essentially determines the energy spread. The small energy spread is achieved at the cost of the intensity of the $\gamma$-ray beam. A typical intensity with the $3 \mathrm{~mm} \mathrm{C} 1$ and 2 mm C2 collimators is $10^{5}$ per second.

\subsection{Flux of the $\gamma$-ray beam}

The flux of the LCS $\gamma$-ray beam was determined with the Poisson-fitting method [10] or so-called pile-up method [11]. The intrinsic accuracy of the flux determination with the method is less than $0.1 \%$.

The Talon laser was operated in the Q-witch mode at the frequency $1 \mathrm{kHz}$ for the neutron-multiplicity sorting. Laser photons with the pulse width $40 \mathrm{~ns}$ and pulse interval $500 \mu$ s collide with electrons with 60 ps pulse width and $2 \mathrm{~ns}$ pulse interval at $500 \mathrm{MHz}$. Twenty electron beam bunches intersect with the laser pulse. It is noted that the LCS $\gamma$-ray beam has the same time structure as that of the laser beam. At the laser power of $1 \mathrm{~W}(1 \mathrm{~J} / \mathrm{s})$ and the electron beam current of $300 \mathrm{~mA}$, a large number of laserphoton and electron pairs $\left(N \sim 10^{26}\right)$ participate in collisions with a small probability $p$, producing LCS $\gamma$ rays. Under this physical condition, the number of photons per $\gamma$ pulse $(n)$ follows the Poisson distribution [12, 13], $P_{m}(n)$,

$$
P_{m}(n)=\frac{m^{n}}{n !} e^{-m},
$$

where $m$ is the average number of photons per $\gamma$ pulse; namely, $m=p N$. We produced LCS $\gamma$ rays with typical values $m \sim 10$.

Figure 4 shows a multi-photon spectrum of LCS $\gamma$ rays at $34 \mathrm{MeV}$ measured with a large-volume $(8 " \times 12$ ") $\mathrm{NaI}(\mathrm{Tl})$ detector in the top-up operation of the storage ring at $200 \mathrm{~mA}$ and the best-fit Poisson distribution constructed from single- and multi-photon responses of the $\mathrm{NaI}(\mathrm{Tl})$ detector. One can see that the multi-photon spectrum is excellently reproduced by the Poisson distribution. 


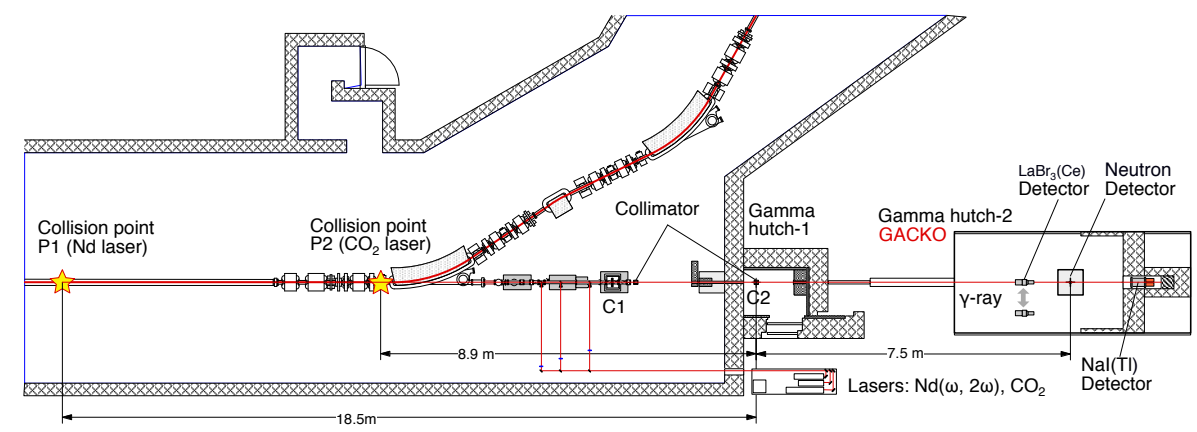

Figure 2. (Color online) $\gamma$-ray beam line of the NewSUBARU facility.

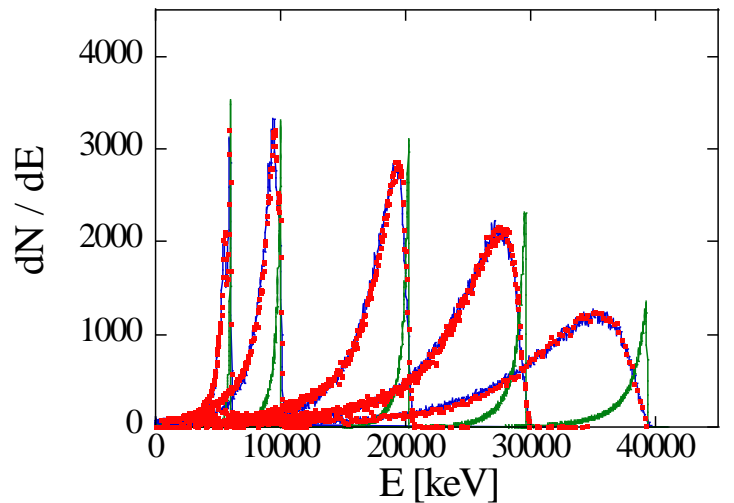

Figure 3. (Color online) Experimental response functions of a $3.5^{\prime \prime} \times 4.0 " \mathrm{LaBr}_{3}(\mathrm{Ce})$ detector to LCS $\gamma$-ray beams (red dots), the best-fit GEANT4 simulations (blue lines) and energy profiles of the incident LCS $\gamma$-ray beam (green lines).

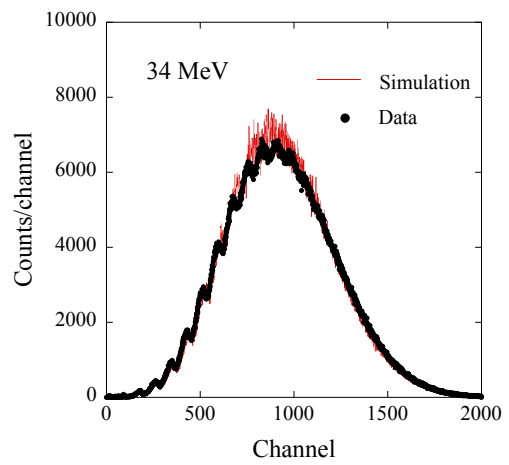

Figure 4. (Color online) Experimental multi-photon spectrum at $34 \mathrm{MeV}$ in comparison with the best-fit Poisson distribution.

Quenching of a multi-photon spectrum was observed for the 40-MeV $\gamma$ rays, which was caused by the saturation at the photomultiplier tube of the $\mathrm{NaI}(\mathrm{Tl})$ detector. However, it is possible to restore the original Poisson distribution with the accurcy $\leq 2.5 \%$ by applying a saturation curve to the quenched spectrum [10].

\subsection{Methodology of direct neutron-multiplicity sorting}

A novel methodology of direct neutron-multiplicity sorting with a flat-efficiency detector was developed for the IAEA-CRP [14]. Partial photoneutron cross sections $\sigma(\gamma, i n)$ with neutron-multiplicity $i$ are determined from the number of $(\gamma, i n)$ reactions, $N_{i}$, by

$$
N_{i}=N_{\gamma} \cdot N_{T} \cdot \sigma(\gamma, \text { in }),
$$

where $N_{\gamma}$ is the number of $\gamma$-rays incident on a target and $N_{T}$ is the number of target nuclei per unit area.

However, the fact that $N_{i}$ is not a direct experimental observable makes the neutron-multiplicity sorting difficult. To understand the difficulty, let us consider measurements of $\sigma(\gamma, i n)$ between $3 n$ and $4 n$ thresholds by using a $\gamma$-ray pulse beam, where neutron-multiplicity $x$ ranges from 1 to 3 . During the $\gamma$-ray pulse interval, one detects photoneutrons with a neutron detector and obtains single $\left(N_{s}\right)$, double $\left(N_{d}\right)$, and triple $\left(N_{t}\right)$ neutron events, depending on the number of neutrons 1,2 , and 3 detected during the pulse interval, respectively.

The single neutron event $N_{s}$ is written by

$$
N_{s}=N_{1} \cdot \varepsilon+N_{2} \cdot{ }_{2} C_{1} \cdot \varepsilon \cdot(1-\varepsilon)+N_{3} \cdot{ }_{3} C_{1} \cdot \varepsilon \cdot(1-\varepsilon)^{2},
$$

where $\varepsilon$ is the neutron-detection efficiency. The first term in Eq. 3 simply means that the $(\gamma, n)$ reaction takes place $N_{1}$ times, each emitting one neutron which is detected with the efficiency $\varepsilon$. The second term means that the $(\gamma, 2 n)$ reaction takes place $N_{2}$ times, each emitting two neutrons one of which is detected with the efficiency $\varepsilon$, while the other is missed with the probability $(1-\varepsilon)$. Finally, the third term means that the $(\gamma, 3 n)$ reaction takes place $N_{3}$ times, each emitting three neutrons one of which is detected with the efficiency $\varepsilon$, while the other two are missed with the probability $(1-\varepsilon)^{2}$.

Similarly, the double and triple neutron events, $N_{d}$ and $N_{t}$, are written by

$$
N_{d}=N_{2} \cdot \varepsilon^{2}+N_{3} \cdot{ }_{3} C_{2} \cdot \varepsilon^{2} \cdot(1-\varepsilon),
$$

and

$$
N_{t}=N_{3} \cdot \varepsilon^{3} .
$$


One can see that if $\varepsilon=1$ (100\% efficiency) theoretically, the number of reactions $\left(N_{1}, N_{2}\right.$, and $\left.N_{3}\right)$ and the experimental observables $\left(N_{s}, N_{d}\right.$ and $\left.N_{t}\right)$ have rigorous oneto-one correspondences, which is however not the case experimentally. Furthermore, the efficiency $\varepsilon$ usually depends on the neutron kinetic energy. For example, the $4 \pi$ paraffin-moderated detector consisting of $48{ }^{10} \mathrm{BF}_{3}$ tubes [15] had 30 - $45 \%$ detection efficiencies over neutron energies up to $5 \mathrm{MeV}$. A neutron-multiplicity sorting performed with the ring-ratio technique $[6,15]$ was modeldependent because the technique which is applied to the experimental observables does not directly determine the average neutron energy for individual $(\gamma$, in $)$ reactions.

When $\varepsilon$ depends on the neutron kinetic energy, Eq. 3 should be written differently. For example, the second term is written by $N_{2} \cdot \varepsilon\left(E_{21}\right) \cdot\left(1-\varepsilon\left(E_{22}\right)\right)+N_{2} \cdot \varepsilon\left(E_{22}\right)$. $\left(1-\varepsilon\left(E_{21}\right)\right)$, using kinetic energies $\left(E_{21}\right.$ and $\left.E_{22}\right)$ of the first neutron and second neutron detected.

An ideal solution for direct neutron-multiplicity sorting is to develop a flat-efficiency neutron detector with $\varepsilon$ which is constant, independent of neutron kinetic energy. Thus, one can solve a set of Eqs. 3, 4, and 5 to obtain $N_{1}$, $N_{2}$, and $N_{3}$ from which partial photoneutron cross sections $\sigma(\gamma, i n)$ with $i=1-3$ are determined.

We have developed a flat-response neutron detector with the detection efficiency of $36.5 \pm 1.6 \%$ over a neutron energy range $0.01-5.0 \mathrm{MeV}$ by optimizing triple-ring configurations of ${ }^{3} \mathrm{He}$ proportional counters embedded in a polyethylene moderator [14]. The efficiency is large enough to obtain the single, double, triple, and quadruple neutron events to determine partial photoneutron cross sections up to $\sigma(\gamma, 4 n)$.

\subsection{Complications in neutron-multiplicity sorting}

Two issues of double firings and secondary $\gamma$ rays need to be handled properly in the neutron-multiplicity sorting. The probability that each $\gamma$ pulse typically containing 10 photons undergoes double firings of photoneutron reactions is small, but not negligible. When a $\gamma$ pulse induces $(\gamma, n)$ reactions twice, we mistakenly regard the doublefiring event as $(\gamma, 2 n)$ reaction. Non-zero $(\gamma, 2 n)$ cross section was obtained immediately below $2 n$ threshold without corrections for double firings, which is regarded as evidence for double firings.

The effect of the electromagnetic interaction (pair production, Compton scattering, and photoelectric absorption) of high-energy $\gamma$-ray beams in a thick target material needs to be taken into account. The interaction produces secondary $\gamma$ rays, which can induce the giant dipole resonance most effectively in the peak $(\sim 13 \mathrm{MeV})$ region governed by the $(\gamma, n)$ channel. We previously assigned extra neutrons produced by the secondary $\gamma$ rays to reaction neutrons of the $(\gamma, n)$ channel associated with the primary $\gamma$ rays [16]. Corrections were made for this effect by means of a GEANT4 simulation, resulting in a significant reduction in ${ }^{209} \mathrm{Bi}(\gamma, \mathrm{n})$ cross section above $30 \mathrm{MeV}$ [17].

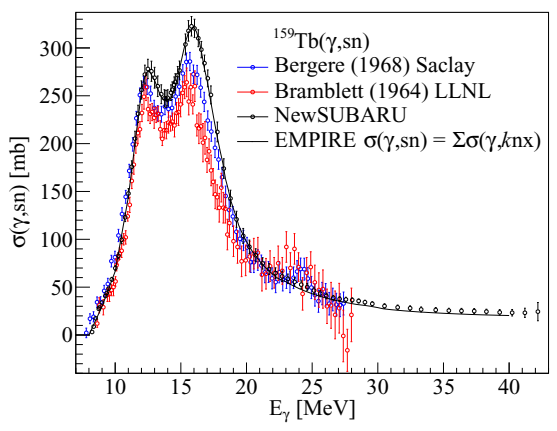

Figure 5. (Color online) The present total photoneutron cross sections, $\sigma(\gamma, s n)$ for ${ }^{159} \mathrm{~Tb}$ compared with the Livermore [22] and Saclay [23] data.

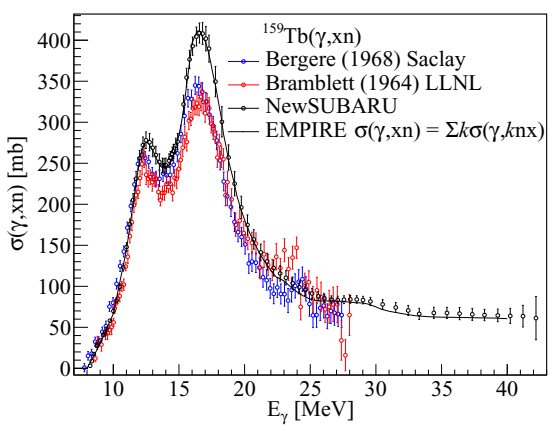

Figure 6. (Color online) The present neutron-yield cross sections, $\sigma(\gamma, x n)$ for ${ }^{159} \mathrm{~Tb}$ compared with the Livermore [22] and Saclay [23] data.

\section{Updated GDR cross sections}

Photoneutron cross sections presented in this paper are classified into partial cross section denoted as $\sigma(\gamma, i n)$, total cross section denoted as $\sigma(\gamma, s n)$, and neutron-yield cross section denoted as $\sigma(\gamma, x n)$. In the present measurement of photoneutron cross sections, only neutrons were detected. This means that the cross section implicitly contains contributions from charged-particle emissions and should read as follows.

$$
\sigma(\gamma, i n)=\sigma(\gamma, \text { in })+\sigma(\gamma, \text { inp })+\sigma(\gamma, \text { in } \alpha)+\ldots
$$

$$
\begin{aligned}
\sigma(\gamma, s n)= & \sigma(\gamma, 1 n)+\sigma(\gamma, 1 n p)+\sigma(\gamma, 1 n \alpha)+ \\
& \sigma(\gamma, 2 n)+\sigma(\gamma, 2 n p)+\sigma(\gamma, 2 n \alpha)+\ldots \\
= & \sum_{i} \sigma(\gamma, \text { in }) .
\end{aligned}
$$

$$
\begin{aligned}
\sigma(\gamma, x n)= & \sigma(\gamma, 1 n)+\sigma(\gamma, 1 n p)+\sigma(\gamma, 1 n \alpha)+ \\
& 2 \sigma(\gamma, 2 n)+2 \sigma(\gamma, 2 n p)+2 \sigma(\gamma, 2 n \alpha)+\ldots \\
= & \sum_{i} i \sigma(\gamma, i n) .
\end{aligned}
$$



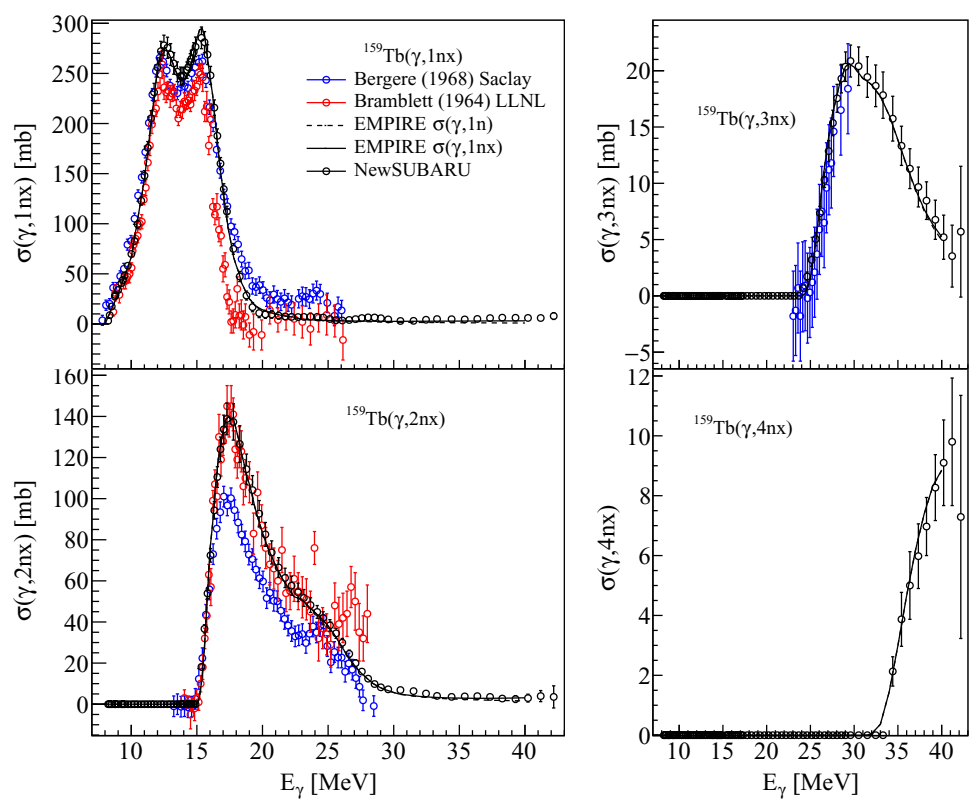

Figure 7. (Color online) The present partial photoneutron cross sections, $\sigma(\gamma$, in $)$ with $i=1-4$, for ${ }^{159} \mathrm{~Tb}$ compared with the Livermore [22] and Saclay [23] data.
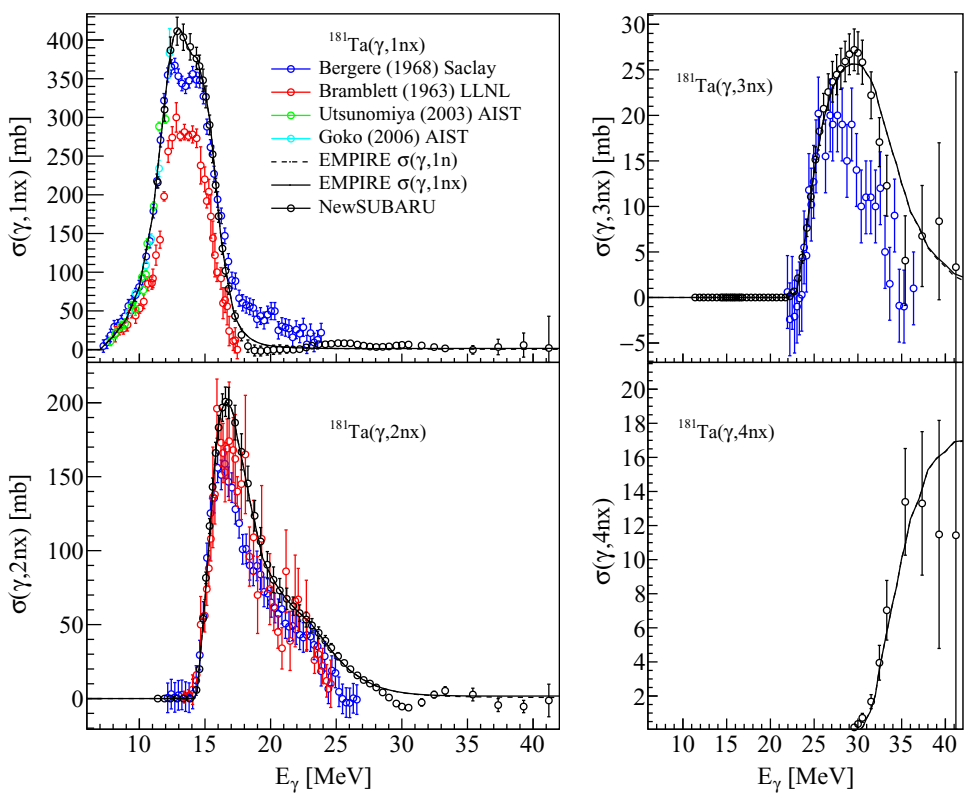

Figure 8. (Color online) The present partial photoneutron cross sections, $\sigma(\gamma$, in $)$ with $i=1-4$, for ${ }^{181}$ Ta compared with the Livermore [24] and Saclay [23] data. The low-energy data [25, 26] are also shown.

\section{$3.1{ }^{159} \mathrm{~Tb}$}

Figures 5, 6, and 7 show total $\sigma(\gamma, s n)$, neutron-yield $\sigma(\gamma, x n)$, and partial $\sigma(\gamma, i n)(i=1-4)$ cross sections for ${ }^{159} \mathrm{~Tb}$ in comparison with the Livermore [22] and Saclay [23] data. The Saclay $\sigma(\gamma, 1 n)$ and $\sigma(\gamma, s n)$ data which are in better agreement with the present data except that the present double-peak cross section is larger than the Saclay cross section. In contrast, the Livermore $\sigma(\gamma, 2 n)$ data are in good agreement with the present data. The Livermore and Saclay data of the neutron-yield cross section which are similar to each other are considerably smaller than the present data especially in the double-peak region.

3.2 ${ }^{181} \mathrm{Ta}$

Figure 8 shows partial cross sections $(\sigma(\gamma, i n), i=1-4)$ for ${ }^{181} \mathrm{Ta}$ in comparison with the Livermore [24] and Saclay 
[23] data. The Saclay $\sigma(\gamma, n)$ data are better agreement with the present data except that the present mono-peak cross section is larger than the Saclay double-peak cross section. The Saclay $\sigma(\gamma, 2 n)$ data are significantly smaller than the present data, while the Livermore data with large error bars seem to lie between the present and the Saclay data. The Saclay $\sigma(\gamma, 3 n)$ data disagree with the present data on high-energy side.

\section{Physics}

The newly measured GDR data are used to update the GDR parameters of the resonance energy, width, and peak cross section which are related to the damping mechanism of GDR and nuclear deformation on the one hand. On the other hand, the data have an impact on physics with the dipole polarizability which is defined by photoabsorption cross section $[18,19]$. Physics involved in the polarizability is the neutron-skin thickness [20] and symmetry energy of the nuclear equation of state [21].

\section{Summary}

We have newly measured partial and total photoneutron cross sections as well as neutron-yield cross sections for 11 nuclei from ${ }^{9} \mathrm{Be}$ to ${ }^{209} \mathrm{Bi}$ with a novel methodology of direct neutron-multiplicity sorting with a flat-efficiency detector and well-controlled $\gamma$-ray beams produced in laser Compton scattering at the NewSUBARU facility. The new data, which underwent evaluations with the EMPIRE code [27] at IFIN-HH (Horia Hulubei National Institute for Physics and Nuclear Engineering), the CCONE code [28] at JAEA (Japan Atomic Energy Agency), and the GLUNF and MEND-G codes[29] at CIAE (China Institute of Atomic Energy), may help to resolve the longstanding discrepancy between the Livermore and Saclay data of partial photoneutron cross sections and, in addition, to investigate the neutron-skin thickness and the symmetry energy of the nuclear equation of state through the dipole polarizibility. These new experimental data are the basis of some of newly evaluated photonuclear data files in the updated version of IAEA photonuclear data library, which will be released in 2019 [30].

\section{References}

[1] Handbook on photonuclear data for applications: Cross-sections and spectra, IAEA Vienna 2000, IAEATECDOC-1178.

[2] V.V. Varlamov, B.S. Ishkhanov, Phys. Part. Nucl. 35, 459 (2004).

[3] V.V. Varlamov, B.S. Ishkhanov, V.N. Orlin, Phys. Atomic Nuclei 75, 1339 (2012).

[4] P. Dimitriou et al., EPJ Web of Conf. 93, 06004 (2015).
[5] P. Dimitriou, "Photonnuclear Data Library and Photon Strength Functions", talk given in this conference.

[6] B.L. Berman, S.C. Fultz, Rev. Modern Phys. 47, 713 (1975).

[7] H. Utsunomiya et al., IEEE Trans. Nucl. Sci. 61, 1252 (2014).

[8] T. Shima and H. Utsunomiya, in Proceedings of the Nuclear Physics and Gamma-ray Sources for Nuclear Security and Nonproliferation, Tokai, Japan, edited T.Hayakawa et al. (World Scientific Publishing, Singapore, 2014), pp. 151-160.

[9] S. Amano et al., Nuclear Inst. and Methods A 602, 337 (2009).

[10] H. Utsunomiya et al., Nuclear Inst. and Methods A 896, 103 (2018).

[11] T. Kondo et al., Nucl. Instrum. Methods A 659, 462 (2011).

[12] T. Kii et al., in Proceedings of the 12th Symposium on Accelerator Science and Technology, ed. Yasushige Yano (The Institute of Physical and Chemical Research (RIKEN)), Wako, Japan, 1999, pp. 484.

[13] H. Toyokawa et al., IEEE Trans. Nucl. Sci. 47, 1954 (2000).

[14] H. Utsunomiya et al., Nuclear Inst. and Methods A 871, 135 (2017).

[15] B.L. Berman et al., Phys. Rev. 162, 1098 (1967).

[16] I. Gheorghe et al., Phys. Rev. C 96, 044604 (2017).

[17] I. Gheorghe et al., Phys. Rev. C 99, 059901(E) (2019).

[18] O. Bohigas, N. Van Gigi, and D. Vautherin, Phys. Lett. B 102, 105 (1981).

[19] A. Tamio et al., Phys. Rev. Lett. 107, 062502 (2011).

[20] P.-G. Reinhard and W. Nazarewicz, Phys. Rev. C 81, 051303(R) (2010).

[21] W. Satuła, R.A. Wyss, and M. Rafalski, Phys. Rev. C 74, 011301(R) (2006).

[22] R.L. Bramblett, J.T. Caldwell, R.R. Harvey, S.C. Fultz, Phys. Rev. 133, B869 (1964).

[23] R. Bergere, H. Beil, A. Veyssiere, Nucl. Phys. A 121, 463 (1968).

[24] R.L. Bramblett, J.T. Caldwell, G.F. Auchampaugh, S.C. Fultz, Phys. Rev. 129, 2723 (1963).

[25] H. Utsunomiya et al., Phys. Rev. C 67, 015807 (2003).

[26] S. Goko et al., Phys. Rev. Lett. 96, 192501 (2006).

[27] M. Herman, R. Capote, M. Sin, et al., Tech. Rep. INDC(NDS)-0603, International Atomic Energy Agency (2013).

[28] O. Iwamoto, N. Iwamoto, S. Kunieda, F. Minato, and K. Shibata, Nucl. Data Sheets 131, 259 (2016).

[29] Z. Zhang, Comm. Nucl. Data Prog. 22 (1999).

[30] T. Kawano et al., submitted to Nuclear Data Sheets (2019); arXiv:1908.00471v1 [nucl-th] 1 Aug 2019. 